sein. Eine weitgehende Vertheilung des Natriums in der ganzen Masse ist nicht nöthig, es genügt, wenn sich in der Absorptionsmasse wenige Stückchen Natriummetall befinden.

\title{
Zum gefahrlosen Eindampfen feuergefährlicher Flüssigkeiten
} verwendet von der Lind ${ }^{1}$ ) einen mit Rost versehenen Gussmantel, in den ein Becher eingesetzt wird. Das Anheizen des Bechers geschieht durch Würfel aus Glühstoff, die man auf den Rost legt und durch einen Bunsen brenner oder durch Uebergiessen mit Spiritus entzündet.

Zur Bestimmung der Erstarrungstemperatur empfiehlt A. A. Shukoff ${ }^{2}$ ) einen neuen Apparat, welcher eine Abänderung de zu kryoskopischen Bestimmungen dienenden Eijkmann'schen Vorrichtung ist. Er besteht aus einem $3 \mathrm{~cm}$ weiten, $11 \mathrm{~cm}$ hohen cylindrischen Gefäss, das in ein anderes $5 \mathrm{~cm}$ weites eingeschmolzen ist. Zwischen beiden Gefässen ist eine Crookes'sche Leere hergestellt, so dass der Raum vom sogenannten Vacuummantel umschlossen ist.

In den Apparat bringt man die geschmolzene Substanz, verschliesst durch einen Kork, in den ein in $1 / 5$ Grade eingetheiltes Thermometer eingesetzt ist, und schüttelt von oben nach unten, hört aber erst auf, wenn der Inhalt deutlich trüb geworden ist. Je nach der Art der Substanz bleibt die Temperatur einige Zeit constant, oder sie fängt an zu steigen, um ein bestimmtes Maximum zu erreichen. Das Steigen ist sehr verschieden and kann bei Fetten bis zu $5^{0}$ betragen.

Ueber den Einfluss der Temperatur auf die Angaben der Saccharimeter, berichtet A. Her zfeld ${ }^{3}$ ).

In Folge der von dem Verfasser und von Wiechmann beobachten Pressungen der zur Controle dienenden festgefassten Quarzplatten bei höheren Temperaturen, sowie von unregelmässigen Fehlern, die von Wiley bezüglich der Quarzkeile beobachtet wurden, hat Herzfeld umfassende Versuche vorgenommen, und zwar sowohl mit den Quarzplatten als auch mit Zuckerlösungen bei verschiedenen Temperaturen. Das Drehungsvermögen der Quarzplatten hatte sich am meisten geändert beim Erwärmen von in einen Ring gekitteten Platten, weniger bei ganz auf eine Glasplatte aufgekitteten.

1) Zeitschrift f. angew. Chemie 1897, \$. 567.

2) Zeitschrift f. angew. Chemie 1899, S. 563 und 603.

3) Zeitschrift des Ver. d. deutschen Zucker-Industrie 49, Heft 516; rom Verfasser eingesandt. 
$\mathrm{Zu}$ seinen Versuchen benutzte $\mathrm{Herzfeld}$ einen eigens zu dem Zwecke construirten Heizapparat, in den das Polarisationsrohr eingelegt wurde. Auf die Construction des Apparates kann ich hier nicht näher eingehen, die Schlussfolgerungen des Verfassers lasse ich im Wortlaut folgen :

1. Es ist nicht möglich, genaue Polarisationen mit Saccharimetern in einem Raum auszuführen, dessen Temperaturnicht seit mindestens 3 Stunden eine constantewar.

2. Es ist nothwendig, in jedem Laboratorium bestimmte Normaltemperaturen innezuhalten, bei denen die Drehung der Quarzplatten zu ermitteln ist, indem die Lösung des Normalgewichtes Zucker bei der betreffenden Temperatur bereitet, deren Drehung bestimmt und danach der Werth der Quarzplatten berechnet wird.

Verfährt man so und hält auch bei der Analyse die Normaltemperatur inne, so ist es möglich, mit Halbschattenapparaten innerhalb sehr weiter Temperaturintervalle richtige Resultate zu erzielen. Doch sind bei stark von der Justirungstemperatur des Apparates abweichenden Arbeitstemperaturen bei Rohuckexpolarisationen für jeden Grad Abweichung rom Hundertpunkt und für jeden Grad Temperaturabweichung die Resultate zur Correctur der Scalenablesung um 0,00036 zu berichtigen. Diese Correctur wird häufig so gering ausfallen, dass man sievernachlässigen kann.

Bei Abweichung von der einmal gewählten Normalarbeitstemperatur kann man nicht erwarten, dass die Polarisationen richtig ausfallen.

3. Die in der Praxis noch vielfach übliche Controle der Apparate lediglich durch Nullpunkteinstellung gestattet zwar mitrichtigen Instrumenten beider Normaltemperatur, für welcheder Apparatjustirtist (17,5 oder $20^{\circ} \mathrm{C}$.) richtige Polarisationen auszuführen, gewährleistet aber keinesfalls die Erreichungrichtiger Resultatebei abweichenden Temperaturen, da mit den letzteren sich auch derWerth der Scala ändert. 
Im Anschluss hieran verweise ich noch auf einen Bericht von F. Strohmer ${ }^{1}$, über die Prüfung von Quarzplatten im Auftrage der Internationalen Commission für eine einheitliche Methode der Zuckeruntersuchung.

Einen aichungsfähigen Polarisationsapparat hat $\mathrm{G} . \mathrm{Bruhn \textrm {s } ^ { 2 }}$ ) construirt.

Die Scala dieses neuen Apparates ist auf dem Quarzkeil, der Nonins auf dem kurzen Gegenkeil eingeätzt oder eingeritzt. Durch diese Anordnung wird eine Verschiebung der Seala gegen den Keił verhindert und die Längenausdehnungen beider durch Wärme sind stets gleich.

Der Apparat ist direct aichungsfähig. Stellt man mittelst des sogenannten Noniusschlüssels den Gegenkeil so ein, dass bei gleicher Beschattung beider Hälften des Gesichtsfeldes der Nullpunkt des Nonius mit dem Nullpunkt der Scala zusammenfällt, so gibt die rom Aichungsamt aufgestellte eventuelle Fehlertabelle der Scala jederzeit den wahren Werth jedes anderen Scalentheiles mit grösster Genauigkeit an. Eine Controle des Apparates durch Quarzplatten, Zuckerlösungen oder ein zweites Quarzkeilpaar fällt fort, die Helligkeit ist eine sehr hohe, weil die 4 spiegehden Flächen der zweiten Keilcompensation nicht vorhanden sind, was sich besonders bei der Untersuchung dunkel gefärbter Flüssigkeiten angenehm bemerkbar macht.

Ein weiterer Vorzug des $\Lambda$ pparates besteht darin, dass die Durchsichtigkeit der Scala und des Nonius dazu benutzt ist, um beide von der Polarisationslampe aus in derselben Weise wie die zu untersuchende Flüssigkeit zu beleuchten, also durch den Apparat hindurch, weshalb die Anwendung einer besonderen Scalenbeleuchtungslampe wegfallen kann. Ein wesentlicher Vorzug der Construction besteht ferner darin, dass Spannungen des Keils oder Pressungen desselben und damit verbundene Drehungsänderungen unmöglich sind. Der Keil ist mit der Scala unverräckbar verbunden und braucht deshalb keine besondere Fassung.

Ein Gefrierapparat für mikroskopische Beobachiungen, den H. Molis $\mathrm{ch}^{3}$ ) construirt hat, besteht aus einem Gefrierkasten um die

1) Oesterreichisch-Ungarische Zeitschrift für Zuckerindustrie und Landwirthschaft 27, Heft 3; vom Verfasser eingesandt.

2) Zeitschrift f. öfentliche Chemie 5, 208.

3) Oesterreichische Chemiker-Zeitung 1, 16. 